\title{
Visualization of Non-Isothermal Liquids Mixing Processes Under the Influence of External Forces
}

\author{
Alexandr Sataev ${ }^{1}$ and Vyacheslav Andreev ${ }^{1}$ \\ ${ }^{1}$ Nizhny Novgorod state technical university, Minin Street, 24, Nizhny Novgorod, 603950, Russia
}

\begin{abstract}
The paper presents a visualization of the mixing processes of non-isothermal flows in the model of a ship's nuclear power plant under static and dynamic modes. The values obtained at the experimental stand served as the database for visualization. It is a four-loop model of the flow part of a ship's nuclear power plant. To create a dynamic mode (oscillations in one plane), the model is placed on a swinging platform. This paper shows the processes occurring during periodic pitching with a period of 4 seconds and amplitude of 15 degrees. The descending annular section of the circulation tract was chosen as the object of research. Then the resulting database was visualized using the 3DFieldPro program, where a color scale was matched to the temperature data and model coordinates, and also with the help of the OpenCV library with a high-level implementation program $\mathrm{C}++$, the characteristic hot/cold vortices (spots), the boundary of the mixing process, its character in static mode (without fluctuations) and dynamic mode were analyzed. The uneven mixing process was noted, especially in the dynamic mode. The resulting tools allow you to quickly and clearly visualize these processes.
\end{abstract}

Keywords

Ship nuclear power plant, OpenCV, dynamic mode, visualization, non-isothermal flow

\section{Introduction}

Currently, there is a tendency in the development of modern methods of obtaining, processing and analyzing data. This is observed both in research activities when processing experimental data and in operator work when tracking current production parameters, for example, in SCADA systems (supervisory control and data acquisition) used in automated process control systems (APCS) [1].

These processes are based on various methods of scientific visualization. They make it possible to analyze the computational results during the calculation in the widest range of both theoretical problems of hydrodynamics and various practical applications [2], [3].

As such a practical application, we have investigated the mixing processes of non-isothermal flows. They significantly determine the parameters of the coolant at the entrance to the core of a nuclear reactor in modes with an incomplete composition of operating equipment and, as a consequence, the thermal engineering state of the core. In addition, we have investigated these processes in relation to ship nuclear power plants. [4], [5]. The entire ship, as well as the reactor plant installed on it, is practically constantly under the influence of various external dynamic forces that affect its spatial orientation, both in normal operation and especially in emergency situations.

\section{Materials and methods}

To date, the main method for studying thermohydraulic processes is still experiment. To simulate the processes of non-isothermal mixing, an experimental stand was made, which is a four-loop model of the flow path of a ship's nuclear power plant. The downward circular section of the circulation tract

GraphiCon 2021: 31st International Conference on Computer Graphics and Vision, September 27-30, 2021, Nizhny Novgorod, Russia EMAIL: sancho_3685@mail.ru (A. Sataev); vyach.andreev@mail.ru (V.Andreev) ORCID: 0000-0003-2294-9877 (A. Sataev); 0000-0002-7557-352X (V. Andreev)

(c) (i) (C) 2021 Copyright for this paper by its authors.

Use permitted under Creative Commons License Attribution 4.0 
(marked with a red frame in Fig. 1B) was chosen as the object of research. As a research method, the method of temperature sensing was applied, which was implemented by installing a grid of thermistors directly on the model wall along the descending annular section. Each thermistor is directly hermetically sealed into the wall of the model around the circumference and in total they make up 5 belts of 8 sensors with a pitch of $50 \mathrm{~mm}$ between the belts. The system for collecting experimental data allows you to receive signals both from a separate belt of temperature sensors, and from all channels in combination with high sampling. A hydraulic simulator in the form of a single elementary cassette is used to simulate the reactor core.

The experiment consisted in the following: a preheated coolant was injected into one of the circulation loops using a pump, and a relatively cold coolant was injected into the other circulation loop, also using a pump. This paper describes the processes of non-isothermal mixing during injection of flows into opposite circulation loops. The temperatures of the injected media are respectively $60{ }^{\circ} \mathrm{C}$ and $20{ }^{\circ} \mathrm{C}$, with the same flow rates $\mathrm{G}=0.6 \mathrm{~m}^{3} / \mathrm{h}$.

The injection into the model can be carried out in any of 4 radially located nozzles, the flow is diverted to the drain through a nozzle located axially on the top cover.

To initiate vibrations in one / two planes, the model is placed on a swinging platform made in the form of a cruciform suspension. The swing unit includes: geared motors (2), bearing supports, frames, crank assemblies, rods with articulated ends. Electric motors, through the crank mechanism, transmit the rotational force to the rod, which in turn drives the model under study on the platform. By changing the radius of the crank, as well as the speed of the electric motor (frequency converter), it is possible to change the amplitude and period of rolling of the model. The model can oscillate in a given plane, like a mathematical pendulum [6].
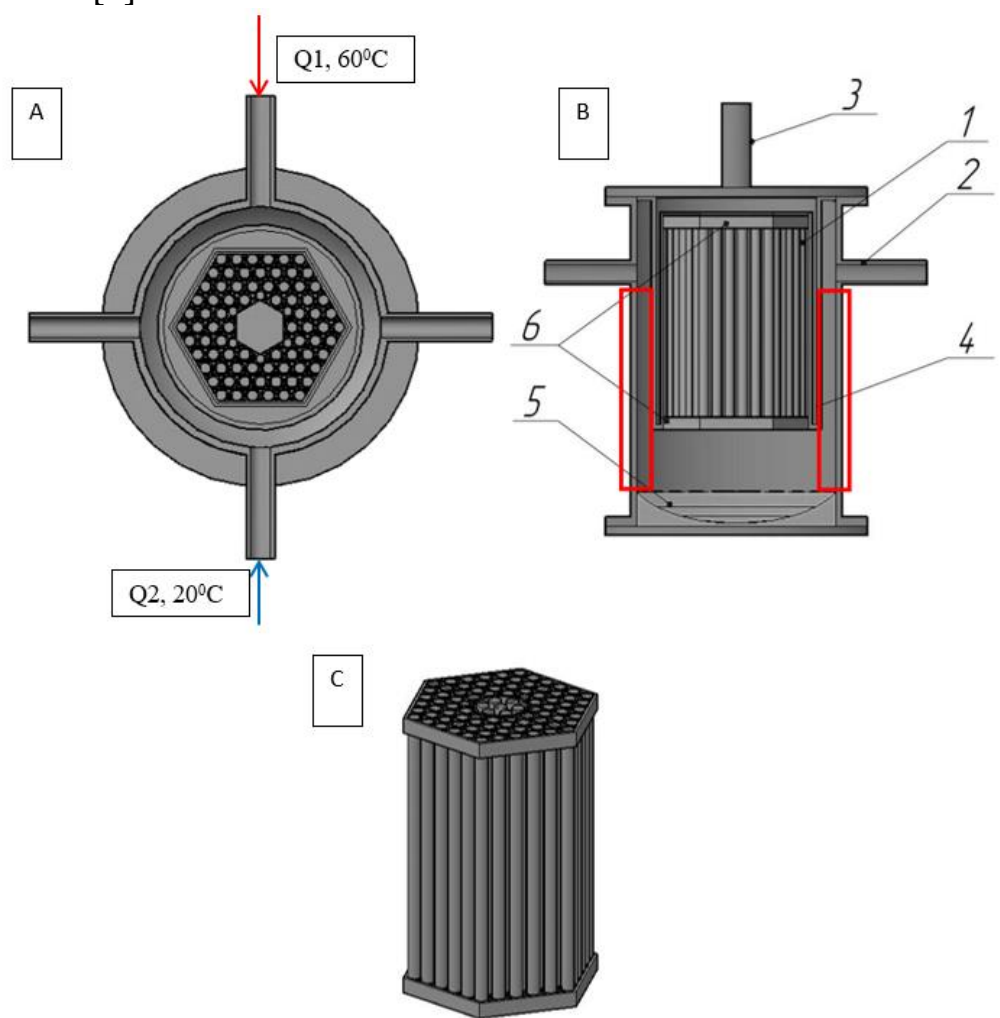

Figure 1: Description of the experimental stand: in fig. A is a sectional view from above; in fig. B - view of the assembled stand, where 1 - fuel assembly simulator model; 2 - coolant supply pipe; 3 - coolant outlet branch pipe; 4- shell; 5- elliptical insert; 6- spacer grilles); in fig. C - model-simulator of fuel assemblies

The choice of parameters for dynamic testing of the model in rolling conditions is justified by the Maritime Register [7]. In addition, it is necessary to separate oscillations by amplitude: with a large 
amplitude ( $>15$ degrees) and small amplitude ( $<15$ degrees), as well as by period: with a large period ( $>10$ seconds) and a small period ( $<10$ seconds). It is obvious that the effect of these oscillations on the described thermohydraulic processes is different. In [8] it is noted that the greatest consequences will be produced by rolling with a short period.

In the experiments shown below in the dynamic mode, the oscillations were carried out in the same plane with a period of $\mathrm{T}=4$ seconds and an amplitude of about 15 degrees. This corresponds to pitching with a small period and the small angle of oscillation.

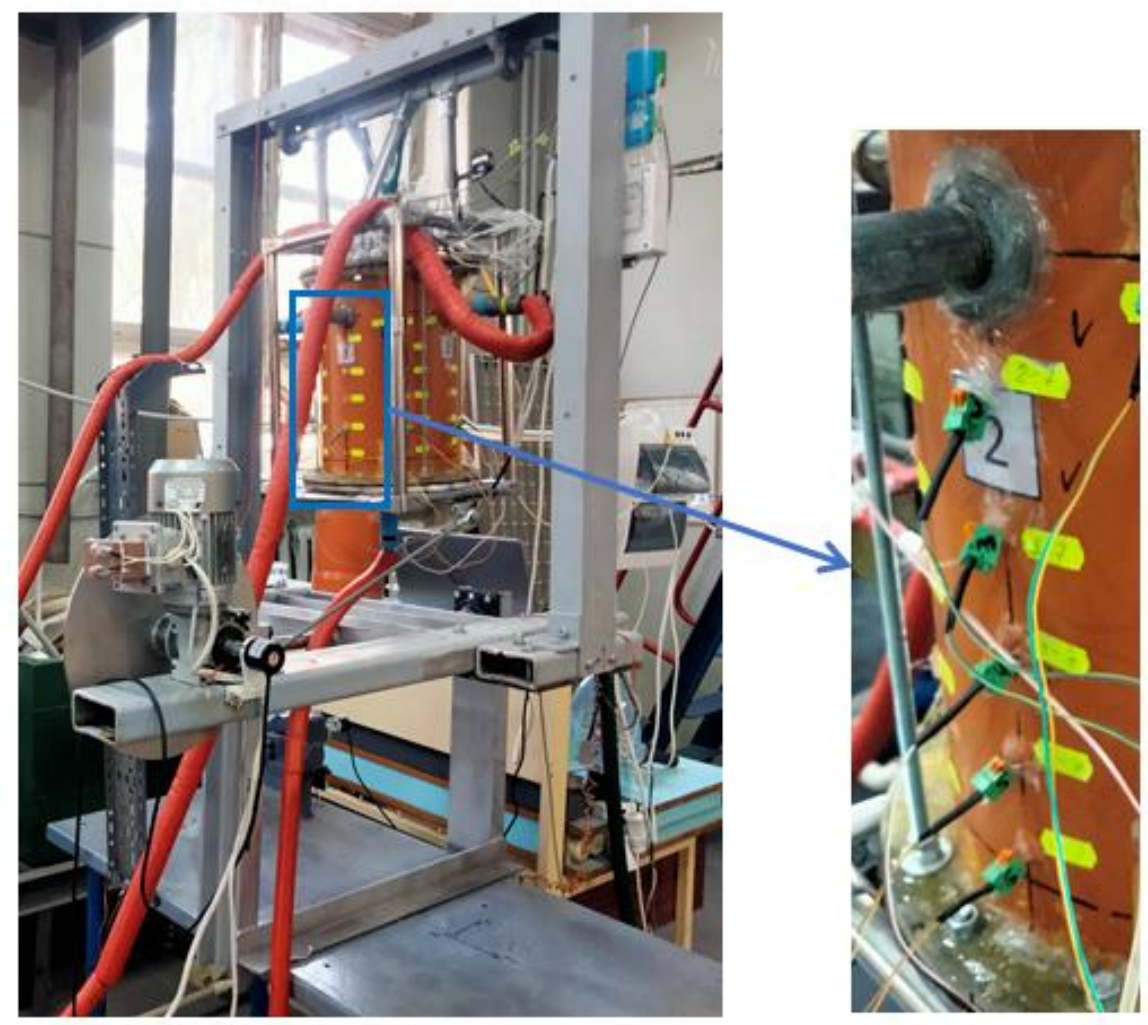

Figure 2: General view of the experimental stand (A local view of the contact groups of temperature sensors is shown separately)

\section{Results and discussion}

In the course of the work, an experimental database of temperatures by characteristic modes was obtained, as well as a database on the position of the experimental model in space, obtained from an angular position sensor. Then the resulting database was visualized using the 3DFieldPro program, where the temperature data and the coordinates of the model were assigned a color scale using the Color Cells function. The Kriging function was used to interpolate the obtained values at other points (Gaussian regression is an interpolation method for which the interpolated values are modeled by a Gaussian process). Figures 3 and 4 show the unfolding of the cylindrical wall of the model together with the coordinate grid, as well as the visualization of the obtained experimental values. Figures 3 and 4 also indicate the nodal points, which show the numbers of the temperature sensors (numbers above the line, where the first number is the number of the level (measurement layer), and the second number is the number of the sensor identified relative to the circle), as well as the current temperature values (numbers below line).

The purpose of further investigation of the obtained images is to analyze characteristic hot / cold vortices (spots), visualize the boundary of the mixing process, and its nature. 


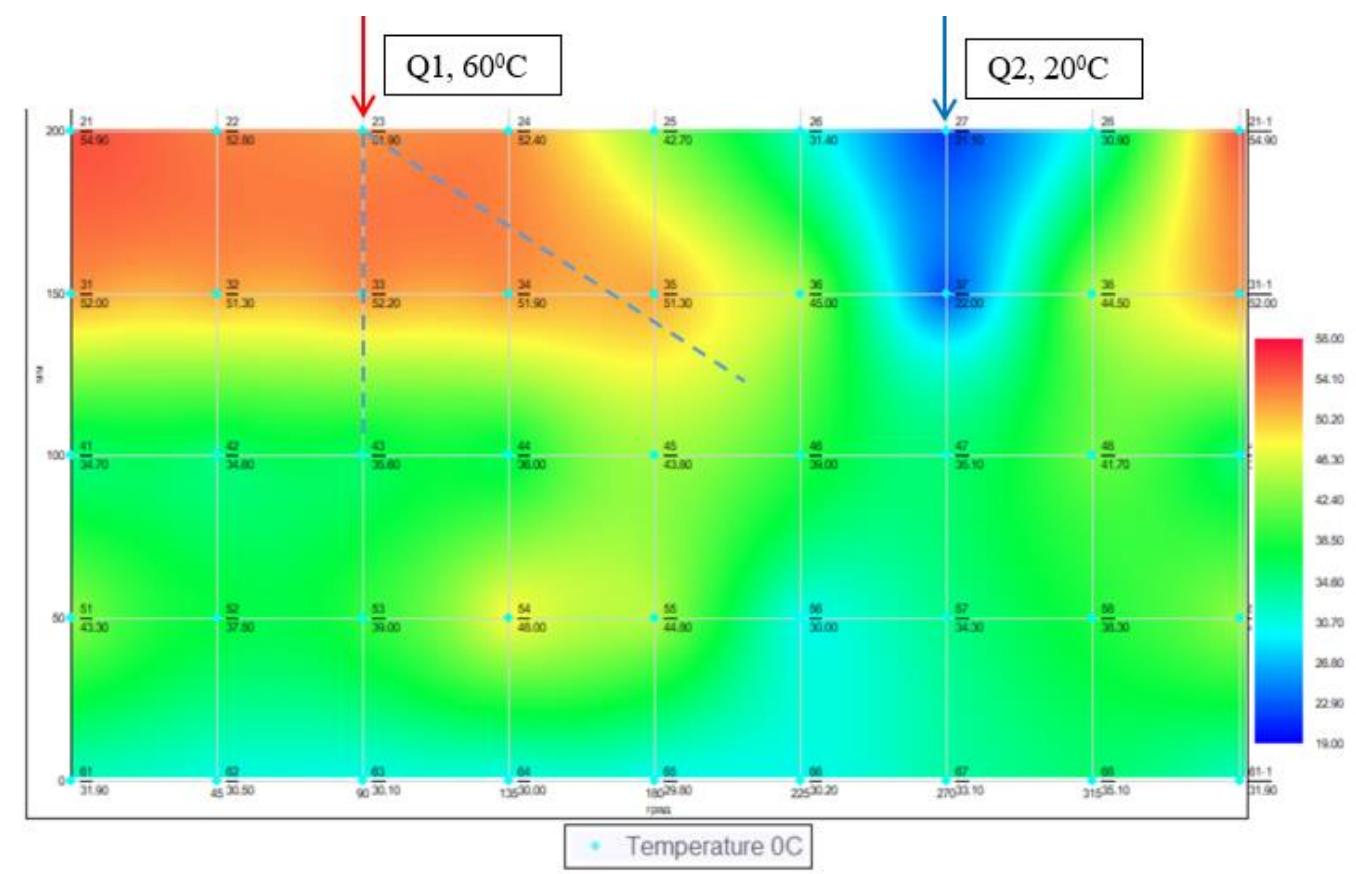

Figure 3: Visualization of the obtained experimental data in the 3DfieldPro program in static mode (without rolling)

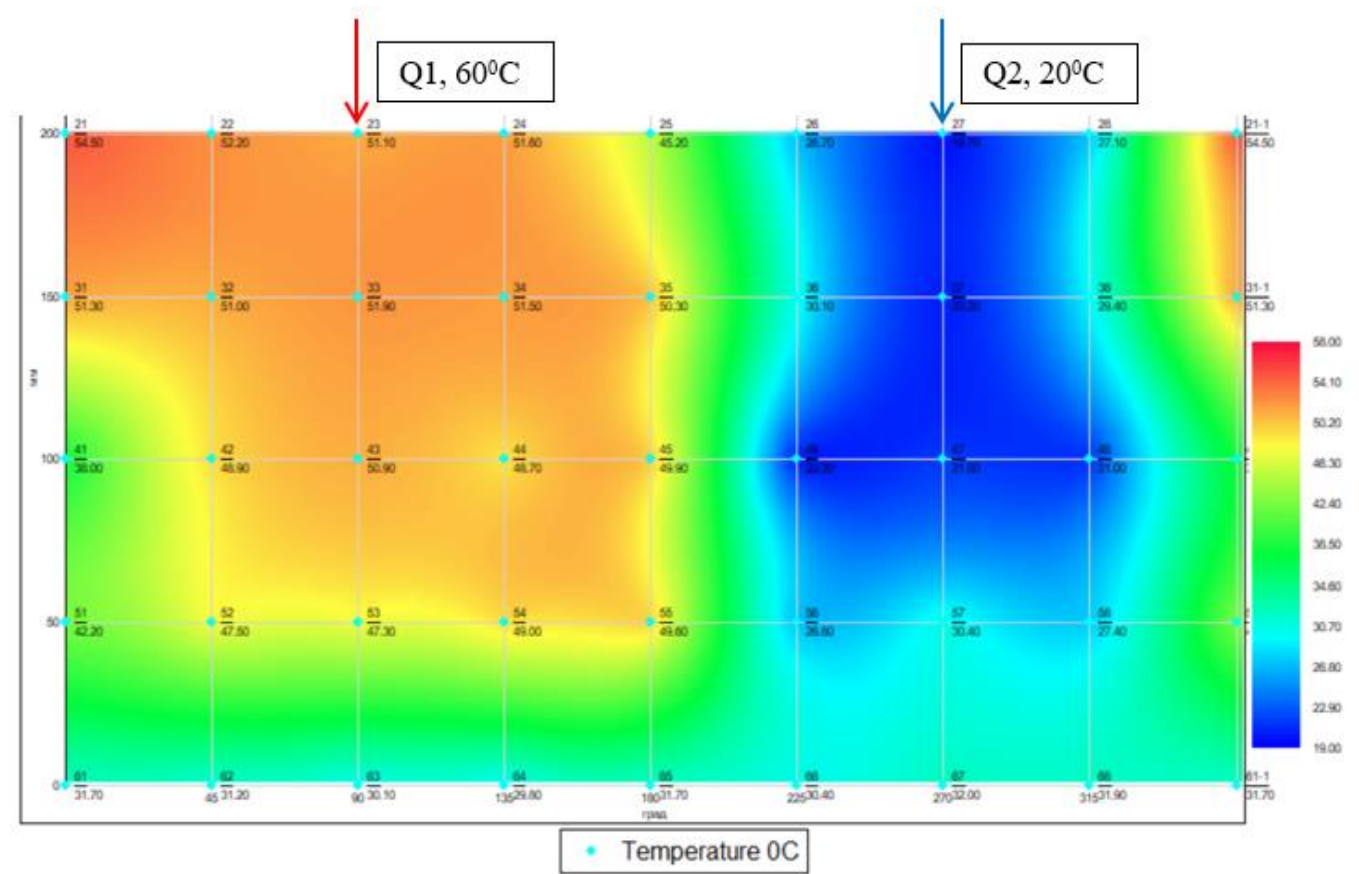

Figure 4: Visualization of the obtained experimental data in the 3DfieldPro program in the dynamic mode (pitching $T=4 \mathrm{sec}$, amplitude of 15 degrees)

To obtain and visualize the boundary of cold and hot regions, a library of computer vision algorithms, image processing and general-purpose numerical algorithms with open source - OpenCV with implementation in the high-level C ++ language - was used [9], [10]. To isolate the boundaries of the hot / cold vortex region, the Canny operator was used to detect characteristic image boundaries.

Boundaries are marked where the gradient of the image is at its maximum value. Based on this, in this way, it is possible to visualize not only the border of the region with the maximum or minimum temperature, but also the region with the maximum gradient. These areas will have a high concentration of ribs, which can be seen in Figures 5 and 6. 


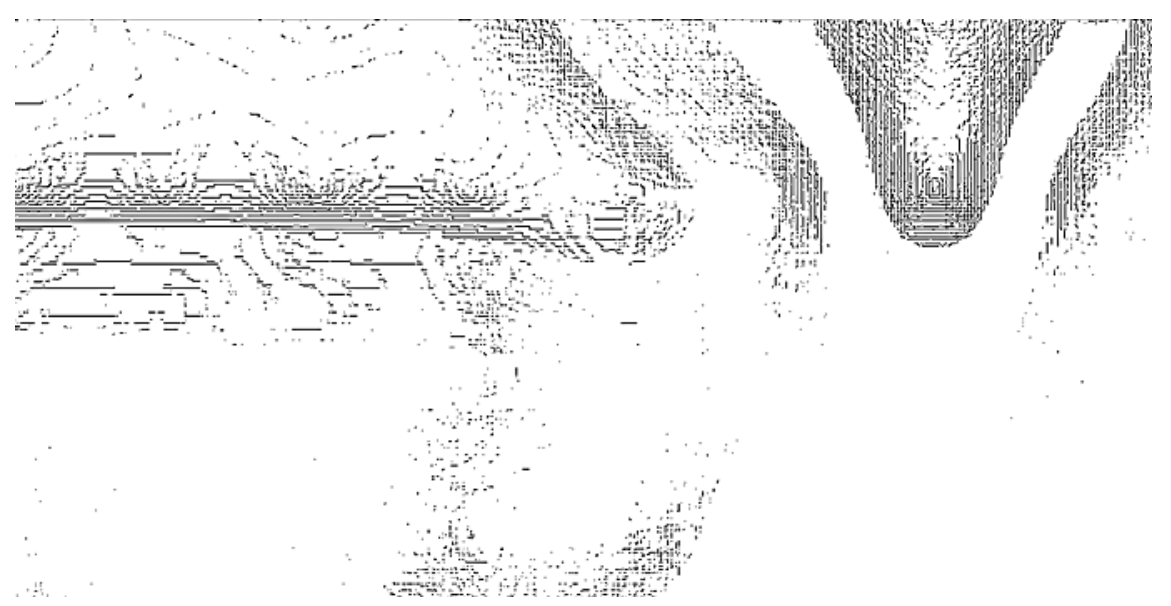

Figure 5: Visualization of the obtained experimental data using the OpenCV library in static mode (without pumping) (inverted)

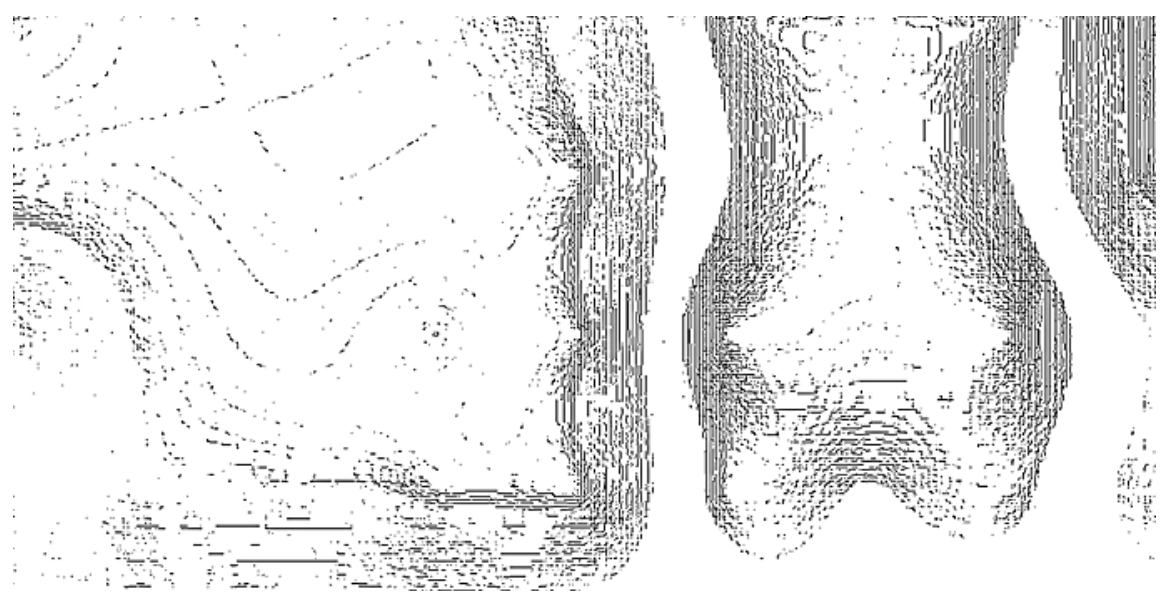

Figure 6: Visualization of the obtained experimental data using the OpenCV library in dynamic mode (pitching $\mathrm{T}=4 \mathrm{sec}$, amplitude of 15 degrees) (inverted)

Based on the images obtained, areas of uneven mixing were identified (a large number of areas with thickened edges). In the static mode, the concentration of hot and cold vortex is observed in the upper part of the model wall; for a hot flow, the flow (swirl) of the flow is noticeably displaced by an angle of about 50 degrees. In the dynamic mode, the concentration (not mixing) of the cold and hot flow occurs directly under the inlet pipe, which is expressed in large areas of high / low temperature.

If we consider it integrally, the mixing occurs more uniformly in the static mode than in the dynamic mode. This confirms the hypothesis about the effect of pitching with a short period, which leads to pulsations of the coolant parameters, which can lead to a limitation of the duration of stay of a nuclear power plant during rolling according to the criteria of fatigue damage to core elements [11].

\section{Conclusion}

As a result of the work, characteristic regions of irregularity were obtained and investigated, which were initiated by the process of non-isothermal mixing. The obtained experimental data were visualized using modern software packages - OpenCV with implementation in the high-level language $\mathrm{C}++$ and 3DFieldPro. A visual and convenient tool for visualization and analysis of process data was obtained. In the future, a more detailed experimental study is planned (with a wide variation of the input conditions) in order to obtain a database of non-isothermal mixing processes under the influence of external forces. 


\section{References}

[1] V.G. Matveykin, S.V. Frolov and M. B. Shekhtman Application of SCADA-systems in the automation of technological processes, Mechanical Engineering (2000).

[2] A. A. Lezhebokov Visualization technologies for applied problems of data mining, Bulletin of the Kabardino-Balkarian Scientific Center of the Russian Academy of Sciences, volume 4 (90), (2019), 14-23. doi:10.35330 / 1991-6639-2019-4-90-14-23.

[3] A. E. Bondarev, V.A. Galaktionov and V.M. Chechetkin Analysis of the development of concepts and methods of visual data representation in problems of computational physics, Zh. Vychisl. mat. and mat. Phys., volume 51 (4), (2011). 669-683.

[4] A. A. Sataev, S. A. Chesnokov, D. I. Novikov and V. V. Andreev Methods of research of thermohydraulic processes under the influence of external dynamic forces, Marine intellectual technologies, volume 1-1 (51), (2021). 23-30. doi:10.37220 / MIT.2021.51.1.003.

[5] A. A. Sataev, V. V. Andreev, A. V. Duntsev, D. I. Novikov and A. A. Berezin Investigation of the influence of gravitational forces on the thermal-hydraulic processes of power plants IOP Conf. Series: Materials Science and Engineering, volume 1035 (2021). doi:10.1088/1757899X/1035/1/012036.

[6] V. V. Andreev and A. A. Sataev Patent for utility model No. 202079 Two-plane test bench for pitching tests and method of its use for testing thermohydraulic models, Filed 03.11.2020, Issued 29.01.2021.

[7] ND 2-020101-112. Russian Maritime register of shipping. Rules for classification and construction of nuclear-powered vessels and floating structures, Saint Petersburg, (2018).

[8] K. N. Chaynikov The general structure of ships, Shipbuilding, (1971).

[9] OpenCV Tutorial C++, URL: https://www.opencv-srf.com/p/introduction.html.

[10] A. Keler Studying OpenCV 3. Development of computer vision programs in $\mathrm{C}++$ using the OpenCV library / A. Keler, G. Bradsky; translated from English by A. A. Slinkin. - Moscow: DMK Press, (2017).

[11] D. G. Kresov On the unification of requirements for the operability of transport reactors during pumping, Shipbuilding, volume 5 (834), (2017). 